\title{
УДК 517.9
}

\section{Non Uniqueness of p-adic Gibbs Distribution for the Ising Model on the Lattice $\mathrm{Z}^{\mathrm{d}}$}

\author{
Zohid T. Tugyonov* \\ Institute of Mathematics \\ Durmon Yuli, 29, Tashkent, 100125 \\ Uzbekistan
}

Received 05.08.2015, received in revised form 24.09.2015, accepted 28.12.2056

In this paper, we show non uniqueness of p-adic Gibbs distribution for the Ising model on the $Z^{d}$. Moreover, we prove that a $p$-adic Gibbs distribution is bounded if and only if $p \neq 2$.

Keywords: Gibbs distribution, Ising model, lattice.

DOI: $10.17516 / 1997-1397-2016-9-1-123-127$.

\section{Introduction}

Real Gibbs measures arise in many problems of probability theory and statistical mechanics. This measure, related to the Boltzmann distribution, generalizes the notion of canonical ensemble. In addition, Gibbs measure is unique measure that maximizes the entropy of the expected energy. But non-archimedean ( $p$-adic) analogue of Gibbs measures have been little studied. It is known that in the case of real numbers concepts of Gibbs measure and Markov random field are identical. But in the $p$-adic case, the class of $p$-adic Markov random fields is wider than the class of $p$-adic Gibbs measures [1]. One of the main problems of physics is to study the set of all $p$-adic Gibbs measures (see e.g. $[1,2]$ ).

Let us present some main definitions from the theory of $p$-adic numbers (see [3-5]). Let $p$ be a prime number. Every rational number $x \neq 0$ can be represented in the form $x=p^{r} \frac{n}{m}$, where $r, n \in \mathbb{Z}, m$ is a positive number, $(n, m)=1$, where $m$ and $n$ are not divisible by $p$. A $p$-adic norm of rational number $x=p^{r} \frac{n}{m}$ is defined as follows

$$
|x|_{p}= \begin{cases}p^{-r}, & \text { if } x \neq 0 \\ 0, & \text { if } x=0\end{cases}
$$

The completion of the set of rational numbers $\mathbb{Q}$ under $p$-adic norm leads to the field of $p$-adic numbers $\mathbb{Q}_{p}$ for every prime $p$. This $p$-adic norm satisfies the strong triangle inequality:

$$
|x+y|_{p} \leqslant \max \left\{|x|_{p},|y|_{p}\right\} .
$$

This property shows that $p$-adic norm is a non-Archimedean norm.

It immediately follows from the strong triangle inequality that

1) if $|x|_{p} \neq|y|_{p}$, then $|x+y|_{p}=\max \left\{|x|_{p},|y|_{p}\right\}$;

2) if $|x|_{p}=|y|_{p}$, then $|x+y|_{p} \leqslant|x|_{p}$; For $a \in \mathbb{Q}_{p}$ and $r>0$ we introduce

$$
B(a, r)=\left\{x \in \mathbb{Q}_{p}:|x-a|_{p}<r\right\} .
$$

*zohidboy@mail.ru

(c) Siberian Federal University. All rights reserved 
The $p$-adic logarithm is defined as

$$
\log _{p}(x)=\log _{p}(1+(x-1))=\sum_{n=1}^{\infty}(-1)^{n+1} \frac{(x-1)^{n}}{n} .
$$

The power series converges for $x \in B(1,1)$. The $p$-adic exponent is defined as

$$
\exp _{p}(x)=\sum_{n=0}^{\infty} \frac{x^{n}}{n !}
$$

and the power series converges for $x \in B\left(0, p^{-1 /(p-1)}\right)$.

Let $x \in B\left(0, p^{-1 /(p .1)}\right.$. Then

$$
\begin{gathered}
\left|\exp _{p}(x)\right|_{p}=1, \quad\left|\exp _{p}(x)-1\right|_{p}=|x|_{p}, \quad\left|\log _{p}(1+x)\right|_{p}=|x|_{p}, \\
\log _{p}\left(\exp _{p}(x)\right)=x, \quad \exp _{p}\left(\log _{p}(1+x)\right)=1+x
\end{gathered}
$$

Let $(X, \mathcal{B})$ be a measurable space, where $\mathcal{B}$ is an algebra of subsets of $X$. The function $\mu: \mathcal{B} \rightarrow \mathbb{Q}_{p}$ is a $p$-adic distribution if for any $A_{1}, \ldots, A_{n} \in \mathcal{B}$ such that $A_{i} \cap A_{j}=\varnothing(i \neq j)$ we have the equality

$$
\mu\left(\bigcup_{j=1}^{n} A_{j}\right)=\sum_{j=1}^{n} \mu\left(A_{j}\right)
$$

A $p$-adic distribution is a measure if

$$
\sup \left\{|\mu(A)|_{p}: A \in \mathcal{B}\right\}<\infty
$$

A $p$-adic measure is a probability measure if $\mu(X)=1$.

Let us consider the set of points $x=\left(x_{1}, \ldots, x_{d}\right), x_{i} \in Z, i=1, \ldots, d$, i.e., the $d$ is dimensional integer lattice $Z^{d}$ with metric $\rho\left(x^{\prime} ; x^{\prime \prime}\right)=\sum_{i=1}^{d}\left|x_{i}^{\prime}-x_{i}^{\prime \prime}\right|$. Two points $x$ and $y$ of the lattice $Z^{d}$ are called nearest-neighbors if $\rho(x ; y)=1$. It is symbolized by $\langle x, y\rangle$.

Let $\mathbb{Q}_{p}$ be the set of $p$-adic numbers and $\Phi=\{-1,+1\}$ is the set of spin values. By standard way we can define the real (see. [6]) or p-adic (see [1]) Gibbs distribution for the Ising model.

For some natural number $n$ the set

$$
M_{n}=\left\{x=\left(x_{1}, \ldots, x_{d}\right), x_{i} \geqslant 0, i=1, \ldots, d: \rho_{1}\left(x, x_{0}\right) \leqslant n\right\}
$$

is called the fundamental quadrate. A point $x \in Z^{d} \backslash M_{n}$ is called nearest-neighbor of the set $M_{n}$ if there exists $y \in M_{n}$ such that $\langle x, y\rangle$, i.e., $\rho(x ; y)=1$. The set of all nearest-neighbors of $M_{n}$ is called the quadrate contour of $M_{n}$ and it is designated as $\partial M_{n}$. Configuration $\sigma$ defined on the set $Z^{d}$ is a function $x \in Z^{d} \rightarrow \sigma(x) \in \Phi$. Restriction of $\sigma$ on any subset $V \subset Z^{d}$ is designated as $\sigma(V)$, i.e., $\sigma(V)=\{\sigma(x), x \in V\}$. The set of all configurations on $M_{n}$ is denoted by $\Omega_{n}$ and the set of all configurations on $Z^{d}$ is denoted by $\Omega$. Hamiltonian of the $p$-adic Ising model is defined as follows:

$$
H(\sigma)=-J \sum_{<x, y>} \sigma(x) \sigma(y), \text { where }|J|_{p} \leqslant \frac{1}{p} .
$$

Conditional Hamiltonian $H_{n}\left(\sigma \mid \sigma^{\prime}\right), \sigma, \sigma^{\prime} \in \Omega$ of the $p$-adic Ising model on $M_{n}$ has the form

$$
H_{n}\left(\sigma \mid \sigma^{\prime}\right)=-J \sum_{\substack{<x, y>\\ x, y \in M_{n}}} \sigma(x) \sigma(y)-J \sum_{\substack{<x, y>\\ x \in M_{n}, y \in \partial M_{n}}} \sigma(x) \sigma^{\prime}(y) .
$$

In this paper, $p$-adic Gibbs distributions for the $p$-adic Ising model on $Z^{d}$ are studied. 


\section{The p-adic Gibbs distributions}

The $p$-adic conditional Gibbs distribution for configurations $\sigma$ on the quadrate $M_{n}$ and $\sigma^{\prime}$ on $Z^{d} \backslash M_{n}$ with the Hamiltonian $H_{n}\left(\sigma \mid \sigma^{\prime}\right)$ is the following $p$-adic number

$$
\mu_{n}\left(\sigma \mid \sigma^{\prime}\right)=\frac{\exp _{p}\left(-H_{n}\left(\sigma \mid \sigma^{\prime}\right)\right)}{\sum_{\sigma \in \Omega_{n}} \exp _{p}\left(-H_{n}\left(\sigma \mid \sigma^{\prime}\right)\right)} .
$$

Let us consider the following configurations:

$$
\sigma^{+}=\{\sigma(x) \equiv 1\}, \quad \sigma^{-}=\{\sigma(x) \equiv-1\} .
$$

For configurations $\sigma^{+}$and $\sigma^{-}$we define two $p$-adic Gibbs conditional distributions on the quadrate $M_{n}$ :

$$
\mu_{n}^{\varepsilon}(\sigma)=\mu\left(\sigma\left(M_{n}\right) \mid \sigma^{\varepsilon}\left(Z^{d} \backslash M_{n}\right)\right)=\frac{\exp _{p}\left(-H_{n}^{\varepsilon}(\sigma)\right)}{z_{n}^{\varepsilon}}, \quad \varepsilon=-,+,
$$

where

$$
\begin{gathered}
H_{n}^{\varepsilon}(\sigma)=-J \sum_{\substack{<x, y>\\
x, y \in M_{n}}} \sigma(x) \sigma(y)-\varepsilon J \sum_{\substack{<x, y>\\
x \in M_{n}, y \in \partial M_{n}}} \sigma(x), \\
z_{n}^{\varepsilon}=\sum_{\sigma \in \Omega_{n}} \exp _{p}\left(-H_{n}^{\varepsilon}(\sigma)\right)
\end{gathered}
$$

Lemma 1. If $p \neq 2,|J|_{p} \leqslant \frac{1}{p}$ then $|H(\sigma)|_{p} \leqslant \frac{1}{p}$.

Proof. Taking into account the strong triangle inequality and using $|\sigma(x)|_{p} \equiv 1$, we obtain

$$
|H(\sigma)|_{p}=\left|J \sum_{<x, y>} \sigma(x) \sigma(y)\right|_{p} \leqslant|J|_{p} \leqslant \frac{1}{p}
$$

Lemma 2. If $p \neq 2$ then $z_{n}^{+}=z_{n}^{-}$and $\left|z_{n}^{+}\right|_{p}=\left|z_{n}^{-}\right|_{p}=1$.

Proof. Let $p \neq 2$. From $H_{n}^{+}(\sigma)=H_{n}^{-}(-\sigma)$ we obtain $z_{n}^{+}=z_{n}^{-}$. Let us consider the following chain of equalities:

$$
\begin{aligned}
& \left|z_{n}^{+}\right|_{p}=\left|\sum_{\sigma \in \Omega_{n}} \exp _{p}\left(-H_{n}^{+}(\sigma)\right)\right|_{p}=\left|\sum_{\sigma \in \Omega_{n}}\left(\exp _{p}\left(-H_{n}^{+}(\sigma)\right)-1\right)+\right| \Omega_{n}||_{p}= \\
& =\max \left\{\left|\sum_{\sigma \in \Omega_{n}}\left(\exp _{p}\left(-H_{n}^{+}(\sigma)\right)-1\right)\right|_{p} ;|| \Omega_{n}||_{p}\right\}=|| \Omega_{n}||_{p}=\left|2^{n^{d}}\right|_{p}=1 .
\end{aligned}
$$

Let us introduce the following notation

$$
H_{n}(\sigma)=-J \sum_{x, y \in M_{n}}<x, y>\sigma(x) \sigma(y) \text {. }
$$

Theorem 1. Let $p \neq 2$. Suppose that there exist limits

$$
\lim _{n \rightarrow \infty} \mu_{n}^{ \pm}(\sigma)=\mu^{ \pm}
$$

Then $p$-adic measures $\mu^{+}$and $\mu^{-}$are different. In addition, for any $r \in N$ a configuration $\sigma$ can be found such that $\left|\mu^{+}(\sigma)-\mu^{-}(\sigma)\right|_{p}=|J|_{p} \cdot p^{-r}$. 
Proof. Let $a_{n}(\sigma)=\left|\mu_{n}^{+}(\sigma)-\mu_{n}^{-}(\sigma)\right|_{p}$.

Using Lemmas 1 and 2, we have

$$
\begin{gathered}
a_{n}(\sigma)=\left|\frac{\exp _{p}\left(-H_{n}^{+}(\sigma)\right)}{z_{n}^{+}}-\frac{\exp _{p}\left(-H_{n}^{-}(\sigma)\right)}{z_{n}^{+}}\right|_{p}= \\
=\frac{1}{\left|z_{n}^{+}\right|_{p}}\left|\exp _{p}\left(H_{n}(\sigma)\right)\left(\exp _{p}\left(J \sum_{\substack{<x, y>\\
x \in M_{n}, y \in \partial M_{n}}} \sigma(x)\right)-\exp _{p}\left(-J \sum_{\substack{x \in M_{n}, y>\\
x \in \partial M_{n}}} \sigma(x)\right)\right)\right|_{p}= \\
=\left|\exp _{p}\left(2 J \sum_{\substack{<x, y>>\\
x \in M_{n}, y \in \partial M_{n}}} \sigma(x)\right)-1\right|_{p}=\left|2 J \sum_{\substack{<x, y>\\
x \in M_{n}, y \in \partial M_{n}}} \sigma(x)\right|=\left|2 J\left(2 d n^{d-1}-2 s^{-}\left(\sigma^{(n)}\right)\right)\right|_{p},
\end{gathered}
$$

where $\sigma^{(n)}=\left\{\sigma(x), x \in \partial M_{n}\right\}$ and $s^{-}\left(\sigma^{(n)}\right)$ is the number of elements of the set

$$
\left\{x \in \partial M_{n}: \sigma(x)=-1\right\} .
$$

Let $n_{0}=\left[\left(\frac{p^{r}}{2 d}\right)^{\frac{1}{d-1}}\right]$. Note that for all $n>n_{0}$ the difference $2 d n^{d-1}-p^{r}$ is a natural number. Then there exists a configuration $\sigma$ such that $\left.\sigma\right|_{\partial M_{n}}=\sigma^{(n)}$ and $s^{-}\left(\sigma^{(n)}\right)=2 n-p^{r}$. For such $n$ the equality $a_{n}\left(\sigma^{(n)}\right)=|4 J|_{p} \cdot p^{-r}=|J|_{p} \cdot p^{-r}$ holds. Therefore we have $\lim _{n \rightarrow \infty} a_{n}(\sigma)=|J|_{p} \cdot p^{-r}$.

Proposition 1. A cardinality of the set $\left\{\sigma:\left|\mu_{n}^{+}(\sigma)-\mu_{n}^{-}(\sigma)\right|_{p}=|J|_{p} \cdot p^{-r}\right\}$ is continuum.

\section{Boundedness of p-adic Gibbs measures.}

In this section we obtain the condition for $p$-adic Gibbs distribution to be a measure (bounded) for the Ising model.

Theorem 2. A p-adic Gibbs distribution for the Ising model is bounded if and only if $p \neq 2$.

Proof. Consider the following expression for $\left|\mu\left(\sigma_{n}\right)\right|_{p}$ :

$$
\begin{gathered}
\left|\mu\left(\sigma_{n}\right)\right|_{p}=\left|\frac{\exp _{p}\left(H\left(\sigma_{n}\right)\right)}{\sum_{\varphi_{n} \in \Omega_{n}} \exp _{p}\left(H\left(\varphi_{n}\right)\right)}\right|_{p}= \\
=\frac{1}{\left|\sum_{\sigma \in \Omega_{n}}\left(\exp _{p}(-H(\sigma))-1\right)+\right| \Omega_{n}||_{p}}=\frac{1}{\left|\sum_{\sigma \in \Omega_{n}}\left(\exp _{p}(-H(\sigma))-1\right)+2^{n^{d}}\right|_{p}}=1
\end{gathered}
$$

if $p \neq 2$.

To prove unboundedness of $\mu$ for $p=2$ one needs only to show that $\mu$ is not bounded on some path in $Z^{d}$. Let $\pi=\left\{\ldots, x_{-1}, x_{0}, x_{1}, \ldots\right\}$ be a path in $Z^{d}$. Marginal distributions on $\pi$ is of the form

$$
\mu_{\pi}\left(w_{n}\right)=P_{w\left(x_{-n}\right)} \prod_{m=-n}^{n-1} P_{w\left(x_{m}\right) w\left(x_{m+1}\right)},
$$


where $w_{n}$ is a configuration on $\left\{x_{-n}, \ldots, x_{0}, \ldots, x_{n}\right\}$ and $P_{i}$ are coordinates of invariant vector of the matrix

$$
\mathbf{P}=\left(P_{i j}\right)=\frac{1}{\exp _{p}(J)+\exp _{p}(-J)}\left(\begin{array}{cc}
\exp _{p}(J) & \exp _{p}(-J) \\
\exp _{p}(-J) & \exp _{p}(J)
\end{array}\right)
$$

We have

$$
\left|P_{i j}\right|_{2}=\frac{1}{\left|\exp _{p}(J)+\exp _{p}(-J)\right|_{2}}=\frac{1}{\left|\left(\exp _{p}(J)-1\right)+\left(\exp _{p}(-J)-1\right)+2\right|_{2}} \geqslant 2
$$

It follows from (5) and (6) that $\mu$ is unbounded for $p=2$.

\section{References}

[1] U.A.Rozikov, O.N.Khakimov, p-adic Gibbs measures and Markov random fields on countable graphs, TMPh, 175(2013), no. 1, 518-525.

[2] U.A.Rozikov, Gibbs measures on Cayley trees, World Sci., Publ. Singapore, 2013.

[3] V.S.Vladimirov, I.V.Volovich, E.V.Zelenov, p-adic Analysis and Mathematical Physics, World Sci., Singapore, 1994.

[4] N.Koblitz, p-adic numbers, p-adic analysis, and zeta-functions, New York, 1984.

[5] A.Yu.Khrennikov, Non-archimedean analysis and its applications, Moscow, Fizmatlit, 2003 (in Russian).

[6] H.-O.Georgii, Gibbs Measures and Phase Transitions, W. de Gruyter, Berlin, 1988.

\section{Неединственность р-адического распределения Гиббса для модели Изинга на решетке $\mathbf{Z}^{\mathrm{d}}$}

\section{Зохид Т. Тугенов}

В данной работе для модели Изинга на $Z^{d}$ мы покажем неединственность р-адического распределения Гиббса. Кроме того, докажем, что р-адические гиббсовские распределение являются ограниченными тогда и толъко тогда, когда $p \neq 2$.

Ключевые слова: распределение Гиббса, модель Изинга, решетка. 